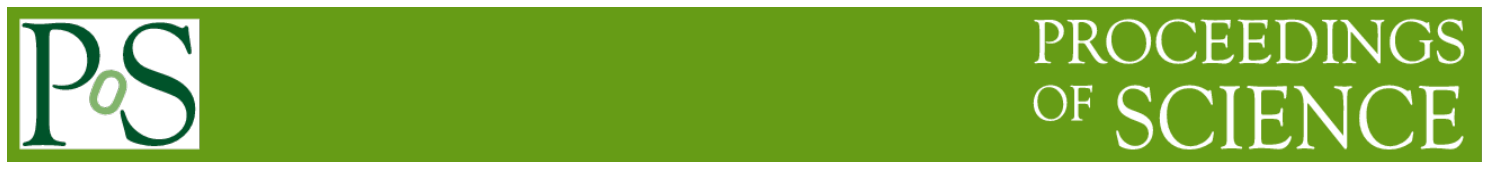

\title{
The role of images in the storytelling of the invisible
}

\author{
Francesca Scianitti ${ }^{1}$ \\ INFN Communications Office \\ Piazza dei Caprettari 70, Rome, Italy \\ E-mail: francesca.scianittiepresid.infn.it \\ Antonella Varaschin \\ INFN Communications Office \\ Piazza dei Caprettari 70, Rome, Italy \\ E-mail: antonella.varaschinepresid.infn.it \\ Vincenzo Napolano \\ INFN Communications Office \\ Piazza dei Caprettari 70, Rome, Italy \\ E-mail: vincenzo.napolanolpresid.infn.it
}

As particle physicists, also science communicators who work on the communication on particle physics are always involved with the traces of something. Images have an increasingly important function in communication through both traditional and new media, and play an even more relevant role in building a good storytelling. Anyway, that's a well-known evidence, particles and forces of nature are not generous in offering images of themselves, and although they determine our universe and even our existence, they are not really familiar objects. That's why building a good storytelling about particles is always a new challenge: that's well-known for those who work in the Communications Offices of Scientific Institutes devoted to research in this field, who have to deal daily with both the constraints of institutional communication and the need to communicate an accessible message in a passionate way in order to reach and involve ever new audiences. Research and experimentation of different ways to communicate have led to the birth of conference-show projects, in which performing arts intertwine with the storytelling of science. But also art exhibitions that, thanks to a proper scenography realized through videos and interactive multimedia installations, seek to create realistic environments. Thus, the interweaving of different communication languages - from the dialogue around science ideas to the use of metaphors, images, cartoons, or artistic multimedia exhibits and performing arts - can lead the public to the discovery of some of the most fascinating ideas of contemporary physics like the discovery of gravitational waves, the search for Dark Matter, but also Albert Einstein's General Relativity or wave-particle duality.

EPS-HEP2017, European Physical Society conference on High Energy Physics

5-12 July 2017

Venice, Italy

\footnotetext{
${ }^{1}$ Speaker

(C) Copyright owned by the author(s) under the terms of the Creative Commons Attribution-NonCommercial-NoDerivatives 4.0 International License (CC BY-NC-ND 4.0). https://pos.sissa.it/
} 


\section{Stories about invisible physics}

When talking about high-energy physics or cosmology, images are rare or unreal. Particles have dimensions that cannot even be imagined; the Universe is made of objects and animated by events, which nature is mostly unperceivable. At all scales, the concepts we use to describe the universe, from the smallest to the biggest one, are hardly comparable with the common ideas that dominate our lives. Nevertheless to build any good story ${ }^{[1]}$ some elements cannot be avoided, like an environment, one or more characters, a plot based on introduction, a rising action, a climax and a final resolution. Furthermore, to create an involvement with the public, all these elements cannot be simply given as a logical sequence of concepts or ideas; they need to be described through their sensorial features: colours, sounds, dimensions and shapes, expressions, speeds, positions in time and space. Talking about physics, some efforts in this direction have been made during the last decade by science communicators all over the world, to find out and experiment new languages making visible what is often not even imaginable.

\subsection{Images of physics}

When building the storyboard of a video, a cartoon or an event, the goal is giving the public the chance to bring with them a good story and a new knowledge. This can be done through very different communication tools and languages: videos - using real or simulated images - animations, drawings and cartoons, multimedia interactive exhibits based on metaphoric interpretations or, again, by building public events during which all these elements can interweave with dialogues and live performances. Though they use different languages, all these instruments aim at giving a visual representation of concepts and objects, and are effective tools to tell any story of physics. The image of a person going through the "Higgs field" while taking mass is very strong and impactful, giving a metaphoric representation of something which name is often well known - after the media impact of the discovery of the Higgs Boson but which, in the most of cases, cannot be explained by people, not even through simple words. In the same way, listening about relativity while seeing an ironic cartoon character behaving in a funny way - when the physics underlined is rigorously drawn and described - can help giving the idea of the sequence of the ideas, by fixing in mind their basic meaning or giving rise to unexpected intuitions about abstract concepts of physics. And even mind images spontaneously created by the public when listening a proper reading or a musical piece are effective ways to create a bridge among concepts and their perception.

\subsubsection{Seeing, listening, feeling: a good environment to understand}

How can images about physics be invented? When looking for a way to explain something it's quite natural to try to find some examples or objects to be used as comparative terms or similitude. That's the natural way to catch for images: looking at all the similarities among the idea that one wants to represent and the things, the environments and the experiences which we are used with. A further step is that of stimulating feelings and perceptions, an effort that usually needs to be supported by languages other then the simple speech, like the artistic language. That's how video-artists can give rise to multimedia installations based on metaphors and capable to build an interactive environment to be experienced by the public, similarly to what happens by looking at an artistic live performance based on a metaphoric interpretation of physics ideas or listening a musical piece whose rhythm and theme are connected with some features of these ideas. 


\section{Public events: physics is on stage}

Since the year 2011 the INFN (Istituto Nazionale di Fisica Nucleare) has started experimenting a new original language to talk about physics. Proposed by the former Head of the INFN Communications Office, Romeo Bassoli, science shows have become in the following years a strong reference point in the outreach activities organized by INFN and having the general public as a target. The first public event of this kind "The Show of the Universe" took place in Naples (Città della Scienza) and was based on the interweaving of the dialogue among scientists with videos and performing arts, like bubble art and aerial juggling with liquid nitrogen spheres. Even the anchorman's figure and his way to behave were properly chosen, in order to be perfectly coherent with the story that was represented on stage. In that occasion the scientists, Fabiola Gianotti, Guido Tonelli and the president of INFN, Fernando Ferroni, were strongly involved in the research on the Higgs Boson, a theme that - after the starting of LHC - was well known but not deeply understood by the public.

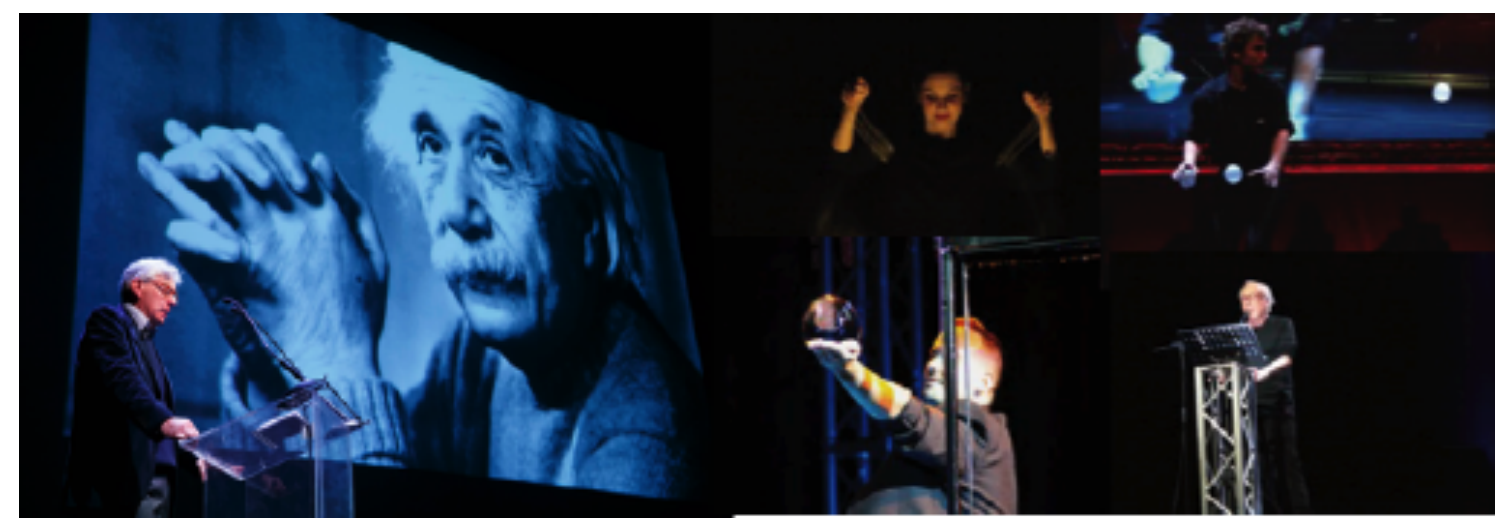

Fig.1: Some pictures of public events organized by INFN in the late years. On the left, "Einstein was right", Rome Auditorium Parco della Musica, 2016. On the right, "What I don't know", Genova Palazzo Ducale, 2014, and Roma Auditorium Parco della Musica, 2015.

After that first event the format has been proposed in other Science Festivals ${ }^{[2]}$ and cultural dissemination contexts, with slight changes time by time in the register, the target public and the goal. If the first experience aimed at catching the interest of families including children, through a storytelling mainly focused on visual performances, in the next editions a major effort has been made to find a clear representation of ideas and contents, in order to give the public the possibility to understand and find an answer to their curiosities. The storytelling has become more coherent with a narrative structure, also supported by videos and animations properly built on it. This is the case of "Quello che non so" (What I don't Know), a conferenceshow about the dark part of both the Universe and the scientists' knowledge, just after the Higgs discovery. The same purpose, with a deeper description of basic concepts about the Universe evolution, was pursued in "Racconto Cosmico" (Cosmic Tale). Based on the ideas of Relativity, the show has been organized in three acts, Space, Matter and Gravity, and the storyboard has been supported by cartoons written by science communicators and drawn by the Italian illustrator Luca Ralli, who succeeded in giving the story an ironic and humorous style, being at the same time coherent with text and the descriptions of physics. 


\section{Exhibitions: imaginative and involving}

Nowadays, scientists' impulse to share and communicate their knowledge is heightened by a further need: how can their work be represented in order that that public opinion and the politicians who decides about research funding can have a picture of its impact on society as much clear and understandable as possible? Technologies in communications give innovative answers to this question. New media, interactive systems, video and digital art, 3D and stereoscopic animations can become the privileged tools to tell the story of science, showing and making the physical description of the world touchable, when it is usually invisible and intangible. Interactive and multimedia systems allow you to imagine and realize unprecedented visual and sensitive metaphors of phenomena or fundamental ideas of the physical world. Furthermore they can create immersive and scenography reproductions of the great experimental apparatuses, to produce spectacular and real-time visuals of the physical phenomena that physicists are looking for.

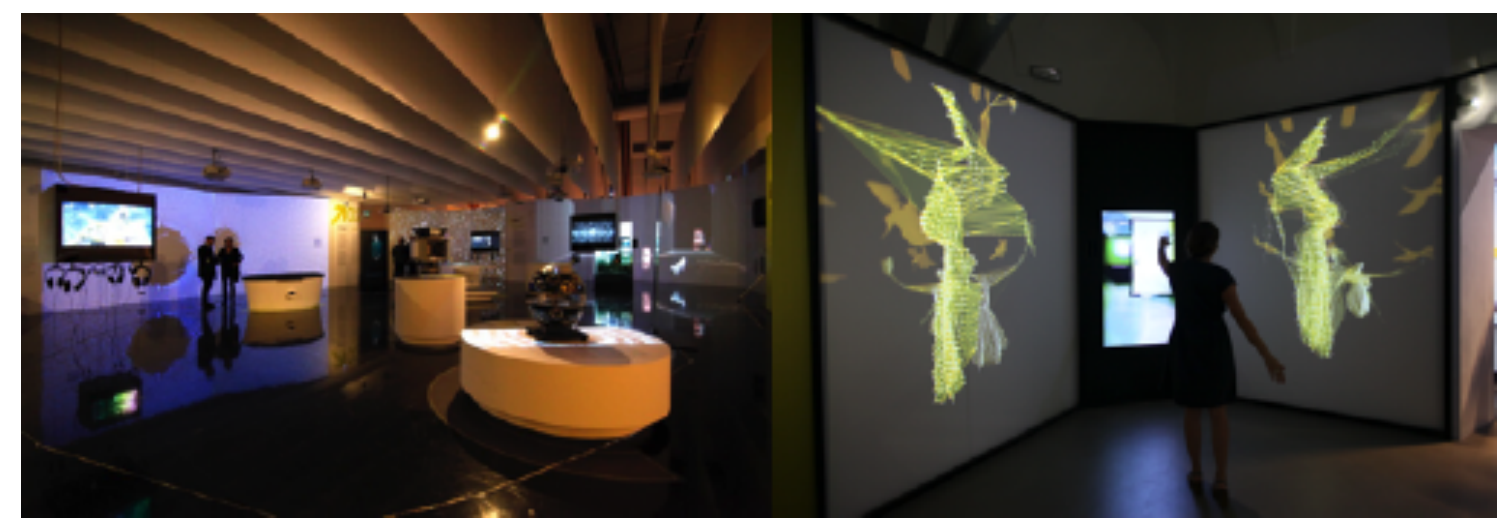

Fig.1: On the left, the exhibition "Beyond the limit", MUSE Trento, 2014-15; on the right, an image of the video art installation "Extradimensions".

Since the year 2009 the INFN has experienced the use of such interactive exhibits to describe particle and astroparticle physics ideas with the exhibition "Astri e Particelle. Le parole dell'Universo" (Stars and Particles. The words of the Universe) ${ }^{[3]}$. People experiencing these artistic multimedia installations have the possibility, for example, to see revealed the uninterrupted flow of cosmic particles around us, or can experience the deformation of a luminous "Spacetime" net under the movements of his own body, embodying the effect caused by the black holes on Cosmo's spacetime structure. Other exhibitions have been developed in the following years and, contemporarily, new interactive exhibits have been designed to explain and visualize physics ideas. With the exhibit "Collisions", for examples, people can virtually speed up their hands with bundles of particles to produce colourful collisions. Playing with their own shadows, people can also "Discover the Invisible", the same invisible content that the technologies that have been developed for particle physics research can discover by looking inside the human body, an artwork or some archaeological finds. More recently, the boundaries of our knowledge have been represented through an exhibition named "Oltre il limite" (Beyond the limit"), which comunicative strenght resided basically on the interactive video-art exhibits named "Spacetime", "Higgs Boson", "Extradimensions", "Universe espansion" and "Wave and Particle". 


\section{Conclusions}

The use of images in disseminating science or talking to the public about researchers' work can be really effective in giving people new tools to understand or perceive the meaning of ideas that are often hard to be communicated in a clear way. Moreover, the use of images can help in building a storytelling of science in which the evolution of the story will be well structured and easily remembered.

Images arise from the contamination of languages that are often based on the collaboration among different professionals: from scientists to science communications, but also new media artists, digital designers, cartoonists, performers, actors. When properly drawn, the story that all these professionals can build together can open a window to unprecedented syntheses among the excellence of science and the artistic expression, as in a real laboratory of new languages.
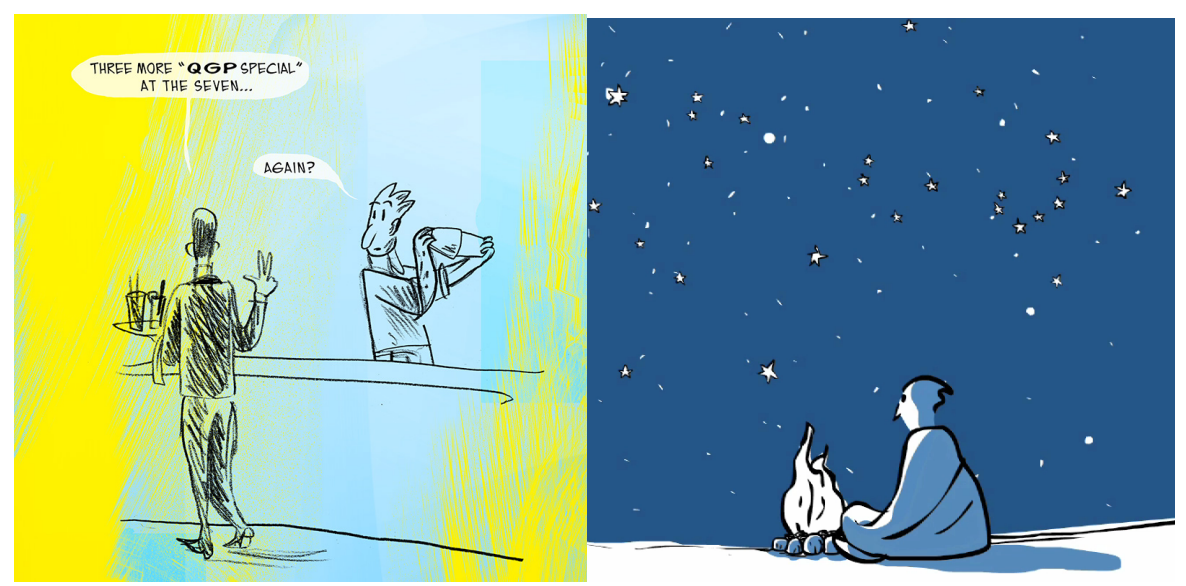

Fig. 3: Two examples of the use of cartoons in the communication of physics.

On the left, a frame of a comics about QGP, drawn for the EPS-HEP 2017 daily newsletter; on the right, a frame of the video cartoon "Space", first of three cartoons on light and Eintein's relativity used to support the storytelling during the public event "Racconto Cosmico".

\section{References}

1. Russell Stannard (2001), Communicating physics through story, Phys. Educ. 36 30, doi: $10.1088 / 0031-9120 / 36 / 1 / 305$

2. Jensen E, Buckley N (2012), Why people attend science festivals: Interests, motivations and selfreported benefits of public engagement with research. Public Understanding of Science, doi: $10.1177 / 0963662512458624$

3. A. A. V. V. (2009), Astri e particelle. Le parole dell'Universo, CODICE Edizioni, Roma, Exhibition Book., ISBN 978-88-7578144-6. 